\title{
Estudio de las percepciones y actitudes del personal de una unidad hospitalaria frente a enfermos terminales
}

\author{
SERGIO BELLO S.*, PATRICIA VERGARA V. **, LORETO O'RYAN S. ***, \\ ANA MARÍA ALFARO B. **** y AMELIA ESPINOSA S. ****
}

Perceptions and attitudes of a respiratory unit staff facing terminal ill patients

In order to promote the humanization of health at the National Institute of Thorax, this work is proposed to make a descriptive and exploratory study of qualitative type with the intention of knowing the functions, perceptions, values and attitudes of the staff of the Respiratory Medical Surgical Unit to faced of the patients who live their process of death in hospital. We are also interested in to identifying strengths, weaknesses and needs felt by the team in caring for these terminal ill patients and the treatment use with the body of the deceased. This information will implement measures that will help to dignify the death in this Unit. It is based on 26 interviews (65\%) of the staff who work in this unit. Interviewees described her/his work as "good" especially in the control of physical symptoms of the patient and family care issues. They admit, however, there are other needs, especially the psychological and spiritual, that they not serve very well. This is the result of a poor training received to resolve high emotional situations and to establish a therapeutic communication with the patient.

Key words: Humanization, end of life care, psychological and spiritual needs of patients.

\section{Resumen}

Enmarcado en el objetivo de promover la humanización de la salud en el Instituto Nacional del Tórax, este trabajo se propuso realizar un estudio descriptivo exploratorio de tipo cualitativo con la finalidad de conocer las funciones, percepciones, valores y actitudes del personal de la Unidad Médico Quirúrgico Respiratorio ante los pacientes que viven su proceso de muerte en el hospital. Nos interesaba también, identificar las fortalezas, carencias y necesidades sentidas por el equipo de trabajo en la atención de estos pacientes terminales y en el tratamiento que se realiza con el cuerpo del fallecido. Esta información permitirá implementar medidas que ayuden a dignificar la muerte en esta Unidad. Se realizaron un total de 26 entrevistas semiestructuradas, correspondientes a un $65 \%$ de los funcionarios que laboran en esta Unidad. Los participantes califican su trabajo como "bueno" especialmente en el control de síntomas físicos del paciente y atención a la familia. Reconocen sin embargo, que hay otras necesidades, especialmente las psicológicas y espirituales que no se atienden con excelencia, como consecuencia de la escasa preparación recibida para enfrentar y resolver situaciones de alto contenido emocional y establecer una comunicación terapéutica con el paciente.

Palabras clave: Humanización, muerte, necesidades psicológicas y espirituales de los pacientes.

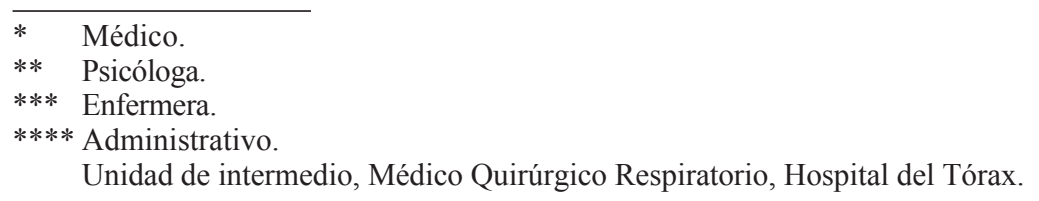




\section{Introducción}

Los avances científicos y tecnológicos en la medicina asistencial y la realidad presente de una sociedad que envejece aceleradamente, demandan hoy importantes modificaciones a la atención sanitaria.

El punto de partida de este cambio de paradigma se expresa en la definición de objetivos de la salud para este tercer milenio. Al objetivo tradicional de curar y prevenir las enfermedades, se incorpora otro de igual importancia y prioridad, que es contribuir a que las personas puedan morir en paz y con dignidad ${ }^{1}$.

En nuestra cultura, se piensa que el mejor lugar para dejar la vida es el propio hogar, en lo posible acompañado y cuidado por los vínculos afectivos cercanos a la persona. Sin embargo, el creciente aumento de las enfermedades crónicas, degenerativas y de patologías de alta complejidad, impiden que un grupo importante de enfermos pueda concretar este anhelo, viéndose obligados a morir en establecimientos hospitalarios.

Por otra parte, la mayoría de los hospitales no están implementados con las condiciones mínimas de bienestar que requieren los pacientes en la fase final de la vida.

En el Instituto Nacional del Tórax (INT), en los últimos tres años, ha fallecido una cifra cercana a 300 enfermos al año, lo que nos da un promedio aproximado de un evento diario. El pronóstico es que estas cifras a futuro podrían ir en aumento, por la gravedad de los pacientes que se reciben.

Si analizamos la trayectoria de nuestra institución, es evidente que la muerte ha sido un tema de menor relevancia, en comparación con los esfuerzos invertidos en curar y prevenir la enfermedad. No obstante, en la actualidad las políticas de salud del Ministerio de Salud ${ }^{2}$ promueven como uno de sus objetivos prioritarios, la humanización de la salud. En este nuevo contexto crece progresivamente la conciencia institucional de que la agonía y la muerte en los hospitales es un tema que debe ser atendido con responsabilidad y excelencia.

Enmarcado en este objetivo la Unidad de Acompañamiento Espiritual del INT se propuso llevar a cabo un proyecto piloto denominado "Dignificación de la muerte", en la Unidad Intermedio Médico Quirúrgico Respiratorio. Esta iniciativa ha sido ideada para desarrollarse en dos fases, una de investigación, que es lo que se presenta a continuación, y una segunda de implementación de mejoras tendientes a otorgar una atención de calidad y excelencia a las personas que fallecen en esta Unidad.

\section{Objetivos del estudio}

- Identificar las principales actividades desarrolladas por cada estamento laboral frente al enfermo que está próximo a la muerte.

- Explorar las fortalezas y debilidades del equipo de salud en la atención del paciente terminal.

- Conocer los recursos materiales e infraestructura que dispone este servicio para satisfacer los requerimientos de los enfermos en su fase de terminalidad.

- Determinar las necesidades de capacitación respecto al manejo del enfermo grave, y el abordaje a las familias.

- Conocer las principales inquietudes, carencias y dificultades que visualiza el personal, en el cuidado del cuerpo fallecido y su traslado a Anatomía Patológica.

- Recoger las sugerencias del personal, que ayuden a que las personas que fallecen en este servicio tengan una muerte digna.

\section{Metodología}

Teniendo en consideración que el objetivo de este estudio era profundizar en las percepciones, motivaciones y actitudes del personal de esta Unidad se estimó pertinente utilizar un diseño de investigación y análisis de la información de tipo cualitativo.

Como técnica de recolección de datos se utilizó una entrevista con dieciocho preguntas abiertas y semiestructuradas. Su ventaja comparativa frente a otras técnicas de investigación, es que permite a los participantes sustentar sus criterios y opiniones generando un amplio y enriquecedor material de análisis.

Las preguntas fueron dirigidas a conocer las funciones y responsabilidades laborales frente al paciente terminal, su satisfacción con el trabajo realizado, la preparación psicológica para atender al paciente terminal, el conocimiento sobre las necesidades del paciente, los sentimientos personales experimentados en este tipo de trabajo y se realizó un ejercicio de imaginería sobre la proyección de la muerte personal. También se preguntó en relación a la valoración que hace cada uno de los funcionarios sobre la atención del paciente premortem y después de su fallecimiento, con sus fortalezas y debilidades. 
Finalmente, se preguntó la opinión sobre demandas de capacitación para brindar una atención de buena calidad y excelencia en estas circunstancias.

Concluido el período de recolección de información, se procedió a transcribir y tabular las respuestas a cada pregunta, segmentándolas por la variable profesión o actividad. Se establecieron cuatro categorías: médicos, enfermeras, técnicos paramédicos y otros (secretaria, kinesiólogo, auxiliares de servicio, nutricionista).

Posteriormente, se analizaron las respuestas, buscando regularidades en cada segmento. En segundo lugar, se compararon los datos entre los grupos para establecer tendencias de opinión, y detectar diferencias en las actitudes de los distintos estamentos. Por tratarse de un estudio exploratorio, se consignaron también algunas respuestas aisladas, que estimamos eran significativas a la luz de la teoría de los cuidados paliativos $^{3}$.

\section{Resultados}

El total de personas que trabajan en este servicio es de cuarenta funcionarios. Se entrevistaron 26 personas, lo que corresponde a un $65 \%$ del universo. Los otros 14 funcionarios no se lograron entrevistar en el período programado para la recolección de datos, en razón de licencias médicas, dificultades de acceso por los horarios debido al sistema de turnos, etc. La distribución en relación a la actividad laboral se observa en la Tabla 1.

\section{Funciones y responsabilidades laborales frente a un paciente terminal}

En las respuestas emitidas, encontramos que algunas funciones son compartidas por el per-

Tabla 1. Número de entrevistados según actividad laboral

\begin{tabular}{lcc}
\hline $\begin{array}{l}\text { Actividad } \\
\text { laboral }\end{array}$ & Funcionarios & $\begin{array}{c}\text { Número de } \\
\text { entrevistados }\end{array}$ \\
\hline Médicos & 6 & 5 \\
Enfermeras & 10 & 7 \\
Técnicos paramédicos & 17 & 9 \\
Auxiliares de servicio & 4 & 2 \\
Nutricionista & 1 & 1 \\
Kinesiólogo & 1 & 1 \\
Secretaria & 1 & 1 \\
Total & 40 & 26 \\
\hline
\end{tabular}

sonal clínico, y otras son específicas y propias de cada profesión o estamento.

Los testimonios del grupo de médicos, indican que predominantemente, focalizan su acción profesional en el tratamiento sintomático. Anotan como otra de sus responsabilidades, supervisar que se provea de adecuado confort al paciente y se atienda sus necesidades. No se registraron acciones orientadas a la familia.

Las enfermeras comentan que su ámbito de acción profesional cubre un mayor espectro de variables de atención. Además de la realización de los procedimientos clínicos para el control sintomático y del cuidado del confort, mencionan entre sus funciones el dar soporte emocional y espiritual que ayude a minimizar los síntomas psicosociales asociados al estado vulnerable del paciente. La información a la familia, es otra de las funciones que asumen con regularidad.

Resulta interesante anotar que el grupo de los técnicos paramédicos reporta unánimemente como una de sus actividades más importantes el proveer de apoyo afectivo y espiritual a los pacientes. Ello es mencionado por sobre incluso los tratamientos de enfermería y el aseo que serían funciones que se les han asignado tradicionalmente.

\section{Satisfacción con el trabajo realizado}

Una apreciable mayoría declara encontrarse aceptablemente satisfecha con la actividad que realiza, en tanto que una tercera parte de los encuestados manifiesta una total satisfacción. Es conveniente subrayar que nadie eligió las categorías insatisfecho o totalmente insatisfecho para describir su actitud en relación al trabajo.

\section{Preparación psicológica para atender al paciente terminal}

Resulta coherente con el nivel de gratificación laboral, que los diferentes estamentos expresen confianza en los propios recursos emocionales para enfrentar las responsabilidades con el paciente y sus familias.

Se observan dos tendencias positivas: Un sector levemente superior al 50\% indica sentirse competente. Los demás entrevistados, se expresan con ciertas reservas, manifestando que sólo estarían relativamente preparados para sobrellevar la carga emocional que implica atender y cuidar a pacientes terminales.

\section{Necesidades de un paciente terminal}

Si bien el control de los síntomas físicos, ocupa un lugar significativo en el trabajo de la 
unidad, los funcionarios, reconocen que las necesidades del paciente, son numerosas y abarcan otras áreas de su personalidad. Indican que para los enfermos en proceso de morir, tan prioritario como la atención farmacológica, es evitar el aislamiento y aliviar el sufrimiento, otorgando un oportuno soporte psicosocial y espiritual.

- "Es importante la parte emocional, conversar, impedir la soledad, darle tranquilidad".

- "Lo espiritual es importante y que esté acompañado de la familia".

Aunque se trata de un porcentaje exiguo, es conveniente dejar constancia que en el grupo de los médicos, hay quienes sostienen que el respeto a la autonomía del enfermo no es una prioridad. Entre las enfermeras también hay personas con un enfoque maternalista que consideran que es preferible eludir ante el paciente detalles respecto de la proximidad de la muerte.

- "No le demuestro que se va a morir, evito que sepan tanto".

- "Mantener la autonomía es la menos relevante de las necesidades".

\section{Sentimientos vivenciados en el trabajo con un enfermo terminal}

Los sentimientos que se generan en el personal frente a un enfermo terminal son en su gran mayoría de índole positiva: afecto, preocupación, protección, compasión y tristeza. En esta misma línea, refieren que experimentan gratificación por la ayuda y los cuidados entregados.

La edad y el tiempo de contacto con el enfermo son variables que modulan estos sentimientos. Confiesan que en mayor medida cuando el paciente es joven los invaden sentimientos dolorosos como la sensación de impotencia, y/o aumento de la ansiedad e incertidumbre.

Se registra una concentración de respuestas más empáticas en el personal paramédico:

- "Depende del paciente. Si es joven, siento pena e impotencia. Si es viejo, tranquilidad".

- "Siento angustia cuando sufre la persona y ansiedad por el entorno familiar".

- "Cuando he visto al paciente más tiempo siento tristeza y pena".

\section{Ejercicio de imaginería: proyección de la muerte personal}

Con la finalidad de establecer los aspectos que el personal considera que ayudarían a morir en paz, se guió a los entrevistados a proyectarse en la inevitable situación en la que serán ellos los protagonistas del proceso de morir.

Aunque se comprueba que hay una variabili- dad de respuestas, encontramos ciertas opciones en las que hay un significativo acuerdo:

Un aspecto altamente valorado en todos los segmentos, es el resguardo de la privacidad. El personal considera que la muerte es un acto íntimo, privado y personal, y no desearían ser expuestos a la mirada pública.

- "Me gustaría morir en una sala individual, no frente a todos, con la mayor privacidad posible".

- "Quisiera que se proteja mi privacidad al menos con biombos".

Morir en casa y no en un establecimiento hospitalario, con la cercanía de sus familiares en el momento de la agonía, es sin duda otra de las condiciones anheladas:

- "Me gustaría morir en mi casa y con mi familia al lado".

- "Quisiera estar de alta, con una buena analgesia, y en mi casa”.

Un factor que consideran indigno y que en ningún caso quisieran vivenciar, es el sufrimiento innecesario ocasionado por la aplicación de tratamientos dolorosos e injustificados y la prolongación de la vida artificialmente.

- "Que no me hagan nada extra. Que me seden si es necesario".

- "Que me permitan morir rápido, sin juntas médicas, sin tratamientos invasivos, sin "charqueos", sin sondas, sin punciones".

Aseguran también que les atenuaría el sufrimiento, el sentirse confortados, consolados y tratados con sensibilidad y afecto.

- “... trato amable; que me atiendan al momento y que no me digan: ya está llamando de nuevo".

- "No me gustaría que me trataran como objeto, que me mostraran sin ropa".

- "No quisiera un trato brusco; reuniones delante de mí y comentarios que confunden; sin toqueteos por alumnos".

Prevalece la convicción de que necesitarían sentirse respetados en su autonomía y en su condición de adultos para tomar decisiones a pesar de la vulnerabilidad física y emocional en la que seguramente se encontrarán.

- "Quisiera que me preguntaran qué necesito, que me respondan a mis preguntas, quiero decidir, que no me miren con lástima".

- "La atención más enfocada a mí que a mis parámetros; que me hablen; que me expliquen lo que me van a hacer". 


\section{Valoración de la atención en el paciente premorten}

Consultados acerca de cómo perciben la atención que ofrecen al paciente antes de fallecer, ninguno de los entrevistados estiman que se ha alcanzado un nivel de excelencia. La mayoría, lo califica como un servicio bueno a muy bueno. El grupo más crítico es el de los médicos quienes indican que la atención oscila entre regular a buena.

\subsection{Fortalezas}

Predomina la idea de que la información oportuna y atenta a la familia cuando el paciente está hospitalizado, es una de las fortalezas del equipo de trabajo. En el grupo de enfermeras esta es la percepción hegemónica. En contraste, los médicos manifiestan que un buen control de síntomas es el aspecto más relevante del trabajo de la unidad.

Se destaca la alta prevalencia de actitudes humanitarias, de respeto y solidaridad con el sufrimiento humano:

- "Hay un sentido de humanidad, de compasión”.

- "Hay preocupación para que el paciente esté cómodo, limpio, sin dolor".

- "Se le brinda el máximo posible: ambiente de respeto, se intenta aislar al paciente".

Otro aspecto relevante es la existencia de una identidad de grupo, atento a responder a los requerimientos de los pacientes y sus familias.

- "Nos conocemos, es un equipo afiatado".

- "Todos dan lo mejor de cada uno para cumplir con sus labores".

- "La atención es de la mejor calidad; se hace lo que más se puede”.

Se fomenta en lo posible la privacidad y el acercamiento de la familia, flexibilizando el horario de visitas para que puedan acompañar al paciente en el proceso final.

- "Facilitamos la visita a familiares más allá de las normas".

- "Se trata de respetar la privacidad; facilitar el acompañamiento familiar".

\subsection{Debilidades del equipo de trabajo}

Aproximadamente un tercio de la muestra total, coincide en indicar que una actitud fatalista y resignada de que "no hay nada más que hacer" es una de las debilidades del grupo entrevistado.

La apatía generada por la idea de que el paciente va morir inevitablemente, y el negarse a aceptar la muerte por que se considera un fracaso, se deja traslucir en dos actitudes extremas:

$\mathrm{O}$ una actitud de evitación y distanciamiento que se traduce en algún grado de abandono al paciente:

- "Hay indiferencia frente al sufrimiento, algo de desidia y abandono".

- "A veces se deja un poco de lado al paciente, falta atención personalizada..."

- "...no siempre hay voluntad de acogida para el sufrimiento del otro".

O la adopción de múltiples e innecesarias acciones médicas para prolongar la vida:

- "Se mantiene con soporte al paciente esquivando la muerte".

- "Se prolonga la vida haciendo sufrir al paciente, exceso de intervención”.

- "Hay encarnizamiento terapéutico, algunos médicos no quieren que se les muera en el turno".

Como otras causas que impiden alcanzar niveles de excelencia, indican:

Las limitaciones de espacio no permiten el aislamiento del paciente, condición que estiman indispensable para alcanzar una muerte digna.

- "Se intenta aislar, pero en la sala es muy difícil conseguirlo".

- "No hay espacio para los familiares".

Falta uniformidad de criterios clínicos en aquellos casos en que no es posible esperar una mejoría del paciente.

- "La atención es buena, pero podría ser mejor, hay criterios disímiles en los casos en que no hay nada que hacer".

- "Falta ponerse de acuerdo sobre necesidades del paciente y familiares. Falta preparar a la familia; hay temor a enfrentar a familiares".

\section{Evaluación de la atención cuando el pa- ciente ha fallecido}

8.1. Cuidado del cuerpo del fallecido

Un segmento apreciable de los funcionarios considera que hay respeto y prolijidad en el trato que se le da al cuerpo del fallecido. Señalan que cuidan especialmente el aspecto del paciente:

- "Se trata de hacer lo mejor para el paciente y su familia, se dan palabras de aliento".

- "Hay preocupación por el rostro para que quede bien, que no quede con la boca abierta, se acomoda, se coloca la prótesis". 
Cabe destacar que en contraposición, las opiniones de algunos médicos son discrepantes y cuestionadores. En aras de la excelencia, vale la pena tomar nota de las razones que este grupo tiene para calificar al servicio de regular a deficiente:

- "Se falta a la dignidad del paciente de manera irrespetuosa ya que se lo deja por largo tiempo abandonado en el pasillo, $y$ en ciertas ocasiones descubierto".

- "La actitud frente al cuerpo no siempre es respetuosa, hay quejas de anatomía patológica sobre el manejo del cuerpo".

- "Hay una falta de estructura mínima para un manejo cuidadoso del cuerpo".

\subsection{Familiares del fallecido}

Si bien el ítem mayoritariamente seleccionado es la calificación intermedia "bueno", el personal siente que es más eficiente en su trabajo con el paciente fallecido que con los familiares, tal es así que nadie eligió la categoría excelente para calificarlo.

Un $20 \%$ evalúa como regular el servicio que se da a los familiares en duelo. Justifican su posición en que no hay ni el lugar ni las normativas que viabilicen la comunicación de la noticia. Se menciona también, como un problema el que no se haya estipulado cual es el miembro del equipo que debe avisar a los parientes. Existe la percepción de que esta falta de claridad entorpece y podría generar más sufrimiento en este delicado momento.

- "No están definidas las responsabilidades, no está normado quién avisa a la familia".

- "Hay atraso en la entrega del certificado de defunción".

- "Los médicos rehúsan dar la noticia, esperan hasta el final para llamar a las familias".

Admiten que no siempre se da apoyo emocional a la familia. Lo atribuyen a falta de tiempo por exceso de trabajo y a la escasa preparación para el manejo de situaciones difíciles, como contener crisis emocionales.

- "No siempre es posible otorgar esta atención, por los horarios y sobrecarga asistencial".

- "No sabemos contener, a veces se prohíbe que lloren (los familiares)".

\section{Traslado al Servicio de Anatomía Patológi- ca del Hospital del Salvador}

Sin duda esta es una de las actividades que recibe los comentarios y objeciones más desvalorizantes. Más de la mitad de los encuestados, piensa que el traslado del fallecido al Servicio de Anatomía Patológica del Hospital del Salvador es poco respetuoso e incluso denigrante. Las críticas se concentran en la falta de camillas adecuadas y en el escaso personal disponible, situación que se agudiza durante la noche.

- "El envío a Anatomía Patológica es francamente desastroso".

- "Las camillas están en mal estado...el paciente se tira en anatomía".

- "No puede ir sólo un funcionario, en las noches falta un auxiliar. Hay que normar esto".

Se cuestiona también que el fallecido se envíe desnudo y desprotegido. Tampoco es infrecuente encontrar en los pasillos, camillas con fallecidos y sin custodia.

- "Se trata como un objeto, se lo lleva desnudo".

- "Sería bueno que no se dejara en los pasillos, se ven camillas abandonadas con un "bulto".

- "Que se lo lleven con bata y pijama. La sábana con la que se tapa el cuerpo, se trasluce”.

Algunos participantes sostienen que el traslado al Servicio de Anatomía Patológica podría perfectamente ser obviado, si el Instituto acondicionara un lugar adecuado para mantener temporalmente el cuerpo.

\section{Demandas de capacitación y actitudes personales que requieren ser mejorados para entregar una atención óptima}

Sin excepción los encuestados, reconocen que hay actitudes personales y profesionales susceptibles de mejora, para alcanzar una mayor eficacia profesional. Las dificultades más frecuentes las atribuyen a la escasa formación que han recibido para sobrellevar la carga emocional de trabajar en un contexto de pérdidas y muerte, y resolver las dificultades que surgen en la comunicación con el paciente.

- "Aprender a enfrentar la familia, a expresarme mejor, a encontrar las palabras adecuadas para poder darles tranquilidad".

- "Calmarme para apoyarlos y no decir cosas que los puedan herir".

- "Ofrecer más apoyo religioso al paciente".

Hay consenso en afirmar que consideran necesaria la capacitación. Entre los temas que estiman oportuno trabajar, sugieren: estrategias de comunicación para dar malas noticias y mejorar la relación de ayuda.

Convienen también, en la necesidad de crear 
espacios terapéuticos, que les permita elaborar las emociones y sentimientos que surgen ante la inminencia de la muerte, como medida preventiva para evitar el agotamiento psíquico y la despersonalización.

El grupo de enfermeras y paramédicos, aspira a que se protocolice y valore las actividades que conlleva dar soporte emocional al paciente, ya que consideran que el acompañamiento es terapéutico, y no debe ser considerado una pérdida de tiempo. Así mismo coinciden en que no es suficiente con la buena voluntad y el sentido común, siendo preciso disponer de un modelo de intervención que mejore la relación de ayuda con el paciente y sus familiares.

\section{Discusión}

Como señalamos en la introducción, este estudio forma parte de un proyecto denominado "Dignificación de la muerte en el Instituto Nacional del Tórax" cuyo objetivo final es mejorar la calidad de la atención realizada en este hospital a los pacientes y familiares en el final de la vida, brindando una atención más humana, que considere todas las dimensiones de la persona enferma (biológica, psicológica, emocional, social, valórica y espiritual).

Del estudio se desprende que tanto los profesionales como el resto del personal, intentan prestar una buena atención al paciente moribundo hospitalizado y se consideran eficientes en algunas áreas como el control sintomático y en brindar adecuado confort al paciente. No obstante, se reconoce que hay defectos y omisiones en la atención de las múltiples complicaciones y requerimientos que presenta el paciente en la agonía y en la muerte. Estas necesidades han sido objeto de variadas publicaciones ${ }^{3-6}$, destacándose los requerimientos en el área emocional, psicológica y espiritual.

En general, aunque con mayor énfasis en el grupo de médicos, predomina un abordaje conservador centrado fundamentalmente en el paciente. No obstante lo anterior, se aprecia en todos los estamentos investigados una progresiva toma de conciencia del ineludible desafío que implica superar el tradicional modelo biomédico centrado únicamente en el cuerpo y la enfermedad. Paulatinamente se instala la idea de que es el ser humano en su integralidad el que requiere ser asistido. Tan importante como atender el cuerpo y el dolor es atenuar el sufrimiento y enfrentar las necesidades psicosociales y espirituales del paciente y su familia.
En este trabajo no se exploraron las percepciones de los pacientes y familiares con respecto a la atención recibida, lo que habría permitido cotejar las opiniones del equipo de salud. Hay evidencia en la literatura internacional ${ }^{7,8}$ y nacional ${ }^{9}$ sobre la insatisfacción tanto de los pacientes y familia como del personal de salud frente a la atención que reciben o que ofrecen en el final de la vida.

Es así como ha surgido la necesidad de mejorar esta atención. Levy et $\mathrm{al}^{10}$, han resumido las principales estrategias que deberían adoptar los profesionales que atienden a estos pacientes para lograr una atención más compasiva y humana del moribundo: desarrollo de una relación personal con la muerte, lograr destrezas comunicacionales como presencia y autenticidad, creación de ambientes adecuados, voluntad de cercanía mostrándose como personas y por último, facilitar las decisiones en el final de la vida. En este sentido valoramos las respuestas obtenidas en nuestro estudio al indagar sobre la proyección de la muerte personal, donde, al cambiar la perspectiva y ponerse en el lugar del enfermo, surgen las respuestas más empáticas y con una mirada más humanizadora de la atención. Cabe destacar que el mayor número de respuestas empáticas las encontramos en el personal paramédico, lo que podría explicarse por la mayor proximidad y tiempo que este estamento permanece con el paciente.

Desde hace relativamente pocos años se ha intentado describir lo que significa realmente una atención de calidad en el final de la vida en el paciente hospitalizado. Para ello se requiere la utilización del método de relación de ayuda o consejería ${ }^{11}$, también conocido como "counselling" de los anglosajones, además del desarrollo de determinadas destrezas, que fueron sistematizadas por Curtis et $\mathrm{al}^{12}$. Para que esto se logre llevar a la práctica se requiere tomar conciencia, especialmente entre los médicos, que esta es un área en que es posible y necesario mejorar las competencias y que es susceptible, como cualquier otra área de la medicina, de entrenamiento dentro de un proceso educacional ${ }^{10,13}$. Esto quedó de manifiesto en los entrevistados cuando concuerdan en la necesidad de fortalecer, por ejemplo, actitudes de autorregulación emocional y entrenamiento en actividades protectoras que eviten el surgimiento de barreras distanciadoras o conductas de sobreimplicación con los pacientes.

Otro problema detectado en las entrevistas a los profesionales es ocasionado por el enfrentamiento de dilemas bioéticos complejos, que sur- 
gen en el trabajo cotidiano en situaciones límites de la vida ${ }^{14}$. La toma de decisiones para limitar los esfuerzos terapéuticos y evitar prolongadas agonías, en pacientes irrecuperables es uno de ellos. Las visiones de los distintos profesionales y la forma de abordar el caso concreto, no siempre coinciden, y no existe una instancia de deliberación para tomar la mejor decisión interdisciplinaria. Se requiere entonces generar espacios de reflexión para analizar los problemas de diversa índole, clarificar las responsabilidades comunes y específicas y establecer protocolos de trabajo para facilitar la toma de decisiones más adecuadas para los pacientes.

Finalmente, la realización de este proyecto contribuyó a que recientemente la institución comprara una camilla especial para el traslado del cuerpo del fallecido, mejorando con ello un aspecto que había sido muy cuestionado por todos los funcionarios.

En la actualidad se está en la etapa de socializar los resultados de la encuesta y de planificar un taller de capacitación a todo el personal en los diversos aspectos del manejo del enfermo al final de la vida. Paralelamente se están realizando las coordinaciones necesarias para normar institucionalmente el cuidado de los pacientes y familias en el final de la vida.

\section{Bibliografía}

1.- CALLAHAN D. Poner límites. Los fines de la Medicina en una sociedad que envejece. Editorial Tricastela. Madrid. 2004.

2.- MINISTERIO DE SALUD. Objetivos Sanitarios 20002010. Serie de Cuadernos de Redes $N^{\circ} 5$. Subsecretaria de Redes Asistenciales. 2002.

3.- ARRANZ P, BARBERO J. Intervención emocional en cuidados paliativos. Modelos y protocolos. Ariel Ciencias Médicas. Madrid 2005.

4.- BAYES R. Intervención psicológica en cuidados paliativos. En http://www.eutanasia.ws/textos/intervencion PCP0706.doc

5.- FRANCA O, FONTES N, GONZÁLEZ M L, NÚÑEZ J, PUIG P, QUEIROLO H, et al. La ayuda (médica) al bien morir. Ars Médica 2005; 11: 87-105.

6.- ASTUDILLO W, MENDINUETA C. Necesidades de los enfermos en el final de la vida. Ars Médica 2005; 11: 137-51.

7.- STEINHAUSER K, CHRISTAKIS N, CLIPP E, MCNEILLY M, MCINTYRE L, TULSKY J. Factors considered important at the end of life by patients, family, physicians and other care providers. JAMA 2000; 284: 2476-82.

8.- SUPPORT. A controlled trial to improve care for seriously ill hospitalized patients. JAMA 1995; 274 : 1591-98.

9.- VIAL P, IBÁÑEZ P, UMAÑA A, REYES M, VIVIANI P, NERVI F. Autoevaluación sobre formación en medicina paliativa en una cohorte de residentes. Rev Méd Chile 2004; 132: 445-52.

10.- LEVY M. End of life care in the intensive care unit: can we do better? Crit Care Med 2001; 29 Suppl: N56-N61.

11.- UNDURRAGA J P, GONZÁLEZ M, CALDERÓN J. Consejería: propuesta de un método de apoyo al paciente terminal. Rev Méd Chile 2006; 134: 1448-54.

12.- CURTIS J R, WENRICH M, CARLINE J, SHANNON $S$, AMBROZY D, PAMSEY P. Understanding physician's skills at providing end-of-life care. Perspectives of patients, families and health care workers. J Gen Intern Med 2001; 16: 41-9.

13.- CURTIS J R. Communicating about end of life care with patients and families in the intensive care unit. Crit Care Clin 2004; 20: 363-80.

14.- CRUCEIRO A. Ética en cuidados paliativos. Editorial Tricastela. Madrid. 2004.

Correspondencia a:

Dr. Sergio Bello S.

J. M. Infante 717

Fono: 5754900

E-mail: sbello@torax.cl 